\title{
Temperatura base, plastocrono e número final de nós no malmequer-do-campo
}

\author{
Base temperature, plastochron and final node number in yellow calendula
}

\author{
Joelma Dutra Fagundes ${ }^{\mathrm{I}}$ Nereu Augusto Streck ${ }^{\mathrm{II}}$ Lindolfo Storck $^{\mathrm{II}}$ \\ Lia Rejane Silveira Reiniger ${ }^{I I}$ Nelson Diehl Kruse ${ }^{\text {III }}$
}

\section{RESUMO}

O malmequer-do-campo (Aspilia montevidensis) é uma planta herbácea perene, nativa do Sul e Sudeste do Brasil, cuja biologia tem sido pouco estudada. O objetivo deste trabalho foi determinar a temperatura base, o plastocrono e o número final de nós de A. montevidensis. Para tanto, foi conduzido um experimento em Santa Maria, RS, com seis épocas de cultivo (20/12/2005, 14/02/2006, 06/03/ 2006, 04/04/2006, 05/05/2006 e 08/06/2006) no delineamento inteiramente casualizado. A unidade experimental foi uma planta cultivada em vasos preenchidos com substrato comercial. $O$ número de nós visíveis foi registrado na haste principal e nas hastes laterais de primeira e segunda ordem. A temperatura base de emissão de nós foi estimada pela metodologia do menor Quadrado Médio do Erro. A soma térmica diária foi calculada a partir do transplante e o plastocrono foi estimado para cada haste. A temperatura base estimada para emissão de nós de A. montevidensis foi de $0^{\circ} \mathrm{C}$. $O$ plastocrono e o número final de nós variam entre hastes $e$ épocas de cultivo, com os menores valores de plastocrono $e$ maiores valores de número final de nós observados na haste principal.

Palavras-chave: graus-dia, desenvolvimento, Aspilia montevidensis, soma térmica.

\section{ABSTRACT}

Yellow calendula (Aspilia montevidensis) is a perenium herbaceous plant native of South and Southeast Brazil that has been little studied about its biology. The objective of this study was to determine the base temperature, the plastochron and the final node number in A. montevidensis. An experiment was conducted in Santa Maria, RS, with six planting dates (12/ 20/2005, 02/14/2006, 03/06/2006, 04/04/2006, 05/05/2006 and 06/08/2006). The experimental design was completely randomized and the experimental unit was one plant grown in pots filled with commercial substratum. The number of visible nodes were measured on the maim stem and on first and second order lateral branches. The base temperature for node appearance was estimated with the mean square error approach. The daily thermal unit was calculated from transplanting and the plastochron $\left({ }^{\circ} \mathrm{C}\right.$ day for the appearance of two successive nodes) was estimated for each stem. The estimated base temperature for node appearance in A. montevidensis was $0^{\circ} \mathrm{C}$. Plastochron and final node number (FNN) vary among stems and planting dates, with the lowest plastochron and the greatest FNN on the MS.

Key words: degree-day, development, Aspilia montevidensis, thermal time.

\section{INTRODUÇÃO}

Aspilia montevidensis (Spreng.) é uma planta herbácea perene pertencente à família Asteraceae, conhecida popularmente como malmequerdo-campo ou malmequer-amarelo (KISSMANN \& GROTH, 1999). Ela desenvolve-se de forma natural ou espontânea nas regiões Sul e Sudeste do Brasil, onde é considerada invasora de campos nativos (BELLINI et al., 1999). A Aspilia montevidensis apresenta propriedades farmacológicas, com possíveis atividades fungicida e antibiótica, além de ação antiinflamatória e antiúlcera (SIMÕES et al., 1989; BELLINI et al., 1999).A grande quantidade e a exuberância de suas flores abrem

IPrograma de Pós-graduação em Agronomia, Universidade Federal de Santa Maria (UFSM), Santa Maria, RS, Brasil.

IIDepartamento de Fitotecnia, Centro de Ciências Rurais (CCR), UFSM. Av. Roraima, 1000, Campus Universitário, 97105-900, Santa Maria, RS, Brasil. E-mail: nstreck1@smail.ufsm.br. *Autor para correspondência.

IIIDepartamento de Defesa Fitossanitária, CCR, UFSM, Santa Maria, RS, Brasil. 
a possibilidade de exploração da espécie como planta ornamental. No entanto, são escassos os trabalhos relacionados à biologia de $\boldsymbol{A}$. montevidensis, os quais podem fornecer informações para definir os tratos culturais e o manejo da espécie com vistas ao seu cultivo (GRAMBONE-GUARATINI et al., 2004).

Nos estudos da biologia das espécies, a quantificação do desenvolvimento vegetal assume particular importância, pois o desenvolvimento referese à diferenciação celular, à iniciação e ao aparecimento de órgãos e se estende até a senescência da planta (HODGES, 1991; WILHELM \& McMASTER, 1995). Como exemplo de parâmetros do desenvolvimento vegetal, tem-se o número de nós acumulados (NN) e o número final de nós (NFN) nas diferentes hastes da planta.

O NN em uma haste pode ser estimado a partir do conhecimento do tempo necessário para o aparecimento de dois nós sucessivos em uma planta. Em dicotiledôneas, como é o caso de $\boldsymbol{A}$. montevidensis, o aparecimento de nós sucessivos na haste é denominado de plastocrono (SINCLAIR, 1984; BAKER \& REDDY, 2001). Como em A. montevidensis há duas folhas simples associadas a cada nó, o NN está diretamente associado à evolução da área foliar da planta, a qual é responsável pela interceptação da radiação solar usada na fotossíntese e pela produção de biomassa pelo dossel vegetal. O NFN é um importante parâmetro de desenvolvimento, pois está diretamente relacionado com a duração do ciclo de desenvolvimento das espécies, de modo que quanto maior o NFN mais longa é a fase vegetativa e, conseqüentemente, mais longo é o ciclo total da cultura (STRECK et al., 2003; STRECK et al., 2006a).

O plastocrono é freqüentemente expresso em termos de soma térmica (STRECK et al., 2005). Os primeiros estudos relacionando o desenvolvimento vegetal com a temperatura do ar foram creditados a René A. F. de Réaumur, por volta de 1730, na França. Essa constante assumida por Réamur foi chamada de soma térmica, com unidade de ${ }^{\circ} \mathrm{C}$ dia (PEREIRA et al., 2002). O conceito de soma térmica é uma medida de tempo biológico que leva em conta o efeito da temperatura nos processos fisiológicos da planta e, portanto, mais realista que o tempo expresso em dias (GILMORE \& ROGERS, 1958; ARNOLD, 1960; RUSSELE et al., 1984; McMASTER \& SMIKA, 1988). O plastocrono, nesse caso, é a soma térmica necessária para o aparecimento de nós sucessivos na haste da planta e tem como unidade ${ }^{\circ} \mathrm{C}$ dia nó ${ }^{-1}$ (BAKER \& REDDY, 2001; STRECK et al., 2005).

Uma das maneiras clássicas de se obter o valor diário de soma térmica é subtrair dos valores de temperatura do ar (geralmente a temperatura média diária do ar) o valor da temperatura base (GILMORE \& ROGERS, 1958; ARNOLD, 1960; McMASTER \& WILHELM, 1997). A temperatura base (Tb) é definida como o valor de temperatura do ar abaixo do qual não ocorre desenvolvimento ou este é tão lento que, para fins de cálculo, pode ser considerado desprezível (BRUNINI et al., 1976; McMASTER \& WILHELM, 1997). Não foi encontrado na literatura registro da Tb para emissão de nós, dado necessário para estimar o plastocrono. Além disso, não foi encontrado registro do NFN de A. montevidensis e, assim, o objetivo deste estudo foi determinar a temperatura base, o plastocrono e o número final de nós de $A$. montevidensis.

\section{MATERIAL E MÉTODOS}

O experimento de épocas de transplante foi conduzido no Departamento de Fitotecnia da Universidade Federal de Santa Maria (UFSM), Santa Maria, RS (latitude 29 $43^{\circ}$ 'S; longitude 53 $42^{\prime}$ W; altitude $95 \mathrm{~m})$. Para tanto, foram realizados transplantes em seis épocas: 1 (20/12/2005), 2 (14/02/2006), 3 (06/03/ 2006), 4 (04/04/2006), 5 (05/05/2006) e 6 (08/06/2006). As cinco primeiras épocas foram conduzidas a campo e a sexta época em casa de vegetação. As plantas foram cultivadas em vasos plásticos pretos, com capacidade de 12l, preenchidos com substrato comercial Plantmax HT. Os vasos foram pintados de branco, visando minimizar a absorção da radiação solar pelas paredes externas e a conseqüente elevação da temperatura do substrato. Os vasos foram espaçados em 1,0m entre si e ficaram dispostos na superfície do solo nos cultivos a campo e a $0,7 \mathrm{~m}$ de altura, sobre uma bancada, no cultivo em casa de vegetação.

Nas cinco épocas do cultivo a campo, foram utilizadas plantas de $\boldsymbol{A}$. montevidensis de ocorrência natural na área do campus da UFSM. Quando o primeiro par de folhas das plantas apresentava cerca de $0,9 \mathrm{~cm}$ de comprimento do limbo, estas foram transplantadas para os vasos, deixando-se duas plantas por vaso. No transplante teve-se o cuidado de retirar um volume de solo sem danificar as raízes a fim de reduzir o estresse de transplante e o comprometimento do crescimento e do desenvolvimento. Para o cultivo em casa de vegetação, foi realizada semeadura no dia 25/05/2006, utilizando-se sementes provenientes da segunda e terceira épocas de cultivo a campo. Aos 14 dias após a semeadura (08/06/2006), quando 50\% das plantas tinham o primeiro par de folhas em torno de $0,9 \mathrm{~cm}$, foi realizado o transplante. Nos cultivos a campo e nos cultivos em casa de vegetação, foram utilizados cinco vasos por época de cultivo, com duas plantas por vaso. 
A adubação dos vasos foi realizada aplicando-se $8 \mathrm{~g}$ de uréia diluídos em 1 litro de água a cada 30 dias. Foram realizadas quatro aplicações em cada época. A irrigação dos vasos foi realizada diariamente ou sempre que necessário de forma a evitar estresse hídrico.

As diferentes épocas de transplante constituíram os tratamentos. O experimento foi um fatorial hierárquico (posições das hastes nas plantas) no delineamento inteiramente casualizado, com sete repetições na época 1 (20/12/2005), nove na época 2 (14/02/2006) e dez repetições nas épocas 3 (06/03/2006), $4(04 / 04 / 2006), 5(05 / 05 / 2006)$ e $6(08 / 06 / 2006)$. Nas épocas 1 e 2, o número de repetições foi menor devido à morte de algumas plantas. Usou-se cada planta como unidade experimental.

A haste principal (HP), as hastes laterais de primeira ordem (HL1) e as laterais de segunda ordem (HL2) foram marcadas em cada planta com arames coloridos. As hastes laterais (HL1 e HL2) foram identificadas em duas posições de sucessão acrópeta na haste principal, na parte basal (primeiro nó da planta representada pela letra B) e na parte apical (último nó da planta, representada pela letra $A$ ), sendo nomeadas HL1B e HL1A para as hastes de primeira ordem e HL2BB, HL2BA, HL2AB e HL2AA para hastes laterais de segunda ordem, respectivamente, em que a última letra representa basal (B) ou apical (A) nas hastes de primeira e segunda ordem (Figura 1).

O número de nós acumulados (NN) na HP e nas hastes laterais de primeira e segunda ordem das plantas marcadas foi contado duas vezes por semana. A contagem iniciou quando as plantas foram transplantadas e terminou quando o botão floral estava visível. O nó foi considerado visível quando o limbo da folha associada ao referido nó apresentava $1,5 \mathrm{~cm}$ de comprimento.

As temperaturas mínima e máxima diárias do ar durante os cultivos a campo foram obtidas a partir das observações realizadas em uma estação meteorológica convencional pertencente ao 8o Distrito de Meteorologia/Instituto Nacional de Meteorologia, localizada a aproximadamente $150 \mathrm{~m}$ do local do experimento. Durante o cultivo em casa de vegetação, foram medidas, diariamente, as temperaturas mínima e máxima diárias do ar, com termômetro de mínima de álcool e termômetro de máxima de mercúrio, instalados no interior de um mini abrigo meteorológico de madeira pintado de branco na altura da bancada ( $0,7 \mathrm{~m}$ de altura).

A soma térmica diária (STd, ${ }^{\circ} \mathrm{C}$ dia) foi calculada por (GILMORE \& ROGERS, 1958; ARNOLD, 1960):
$\mathrm{STd}=[(\mathrm{Tmax}+\mathrm{Tmin}) / 2-\mathrm{Tb}] .1 \mathrm{dia}$

em que Tmax é a temperatura máxima diária do ar $\left({ }^{\circ} \mathrm{C}\right)$, Tmin é a temperatura mínima diária do ar $\left({ }^{\circ} \mathrm{C}\right)$ e Tb é a temperatura base $\left({ }^{\circ} \mathrm{C}\right)$.

A soma térmica acumulada (STa, ${ }^{\circ} \mathrm{C}$ dia) a partir do transplante foi obtida pelo somatório das STd: $\mathrm{STa}=\Sigma \mathrm{STd}$

A fim de estimar a Tb para emissão de nós em A. montevidensis, foi utilizada a metodologia do menor Quadrado Médio do erro (QMe) da regressão entre o NN e STa a partir do transplante (SINCLAIR et al., 2004), utilizando-se dados do NN da HP coletados nas cinco épocas de cultivo a campo. Foram obtidas equações de regressão linear simples a partir dos valores do NN em função dos valores de STa para todas as plantas nas cinco épocas a campo, assumindo-se valores de $\mathrm{Tb}$ variando de zero a $20^{\circ} \mathrm{C}$, com incremento de $0,5^{\circ} \mathrm{C}$. Para cada planta, o valor de Tb que produziu o menor valor de QMe foi considerado o valor mais adequado de Tb. (SINCLAIR et al., 2004).

Para cada haste marcada, obteve-se a regressão linear simples entre os valores de NN na HP, nas HL1 (basal e apical) e nas HL2 (basal e apical) e os valores de soma térmica acumulada (STa) a partir do transplante utilizando-se a Tb estimada. O plastocrono $\left({ }^{\circ} \mathrm{C}\right.$ dia nó $\left.{ }^{-1}\right)$ em cada haste foi estimado como o inverso do coeficiente angular da regressão linear entre NN e STa (BAKER \& REDDY, 2001; STRECK et al., 2005). Nas hastes principal e nas laterais de primeira e segunda ordem marcadas, foi também contado o número final de nós (NFN) após o aparecimento do capítulo nestas hastes.

A análise de variância foi aplicada para verificar a diferença entre as médias e foi aplicado o teste Diferença Mínima Significativa - Teste t (DMS) em nível de 5\% de significância. O software estatístico utilizado para analise de dados foi o aplicativo computacional científico NTIA(EMBRAPA, 1997).

\section{RESULTADOS E DISCUSSÃO}

Com a adubação de $8 g$ de uréia diluídos em 1 litro de água a cada 30 dias, não foram observados sintomas de deficiência de nutrientes durante o ciclo de desenvolvimento das plantas.

As condições meteorológicas foram distintas para as seis épocas de cultivo nos dois ambientes utilizados (campo e casa de vegetação) durante o ciclo de desenvolvimento das plantas. A média da temperatura média diária do ar foi $23,6^{\circ} \mathrm{C}$ $22,6^{\circ} \mathrm{C}, 18,5^{\circ} \mathrm{C}, 16,6^{\circ} \mathrm{C}, 15,9^{\circ} \mathrm{C}$ e $20,4^{\circ} \mathrm{C}$ nas épocas de transplante 1 (20/12/2005), 2 (14/02/2006), 3 (06/03/ 2006), 4 (04/04/2006), 5 (05/05/2006) e 6 (08/06/2006), 


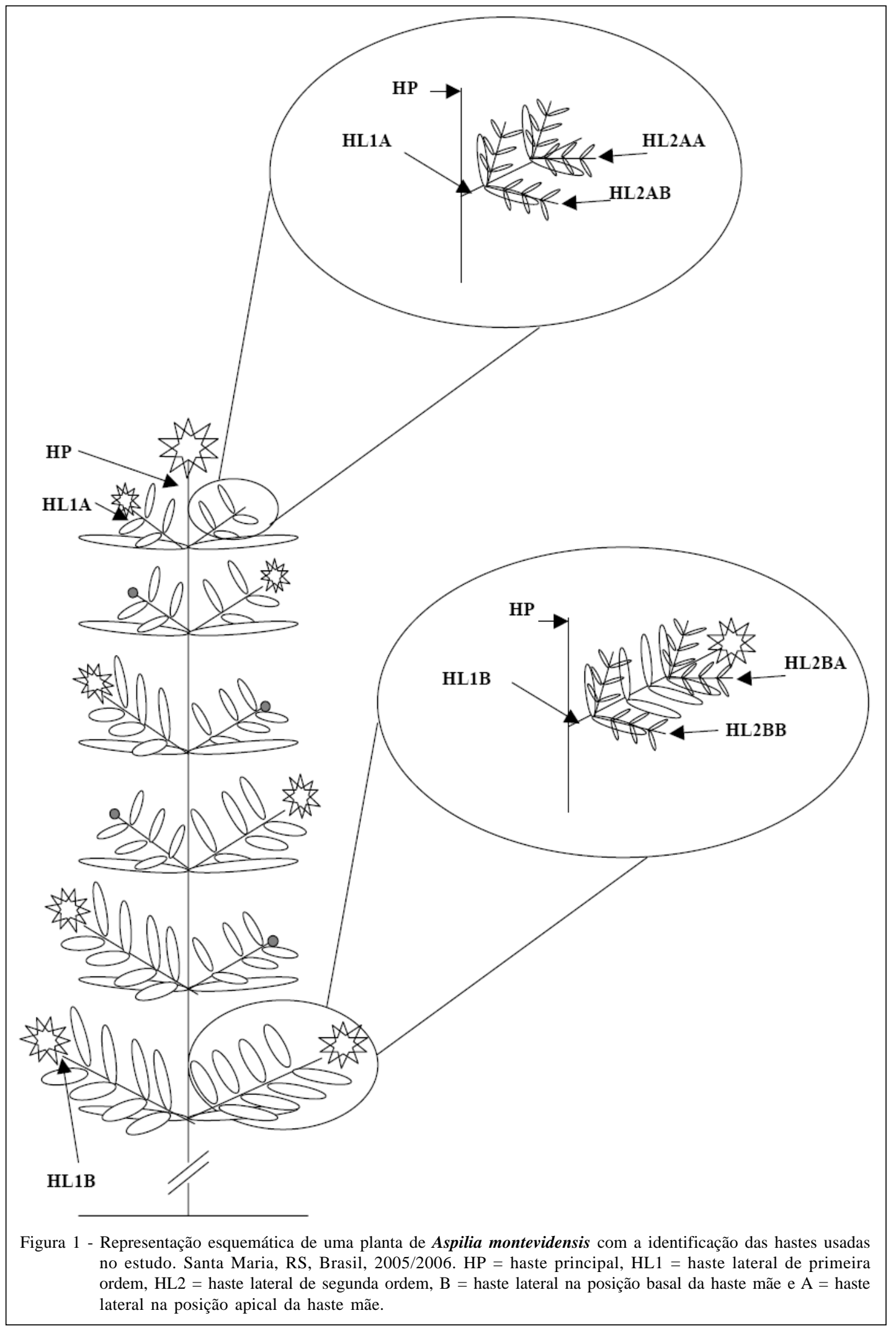

Ciência Rural, v.38, n.9, dez, 2008. 
respectivamente. A menor temperatura mínima absoluta do ar foi de $-0,2^{\circ} \mathrm{C}$, ocorrida em $01 / 08 / 2006$, e a maior temperatura máxima absoluta do ar foi de $39,2^{\circ} \mathrm{C}$, ocorrida em 14/10/2006. Estas condições distintas de temperatura do ar às quais as plantas foram expostas são importantes em estudos bioclimáticos das culturas e necessárias para a estimativa da temperatura base (SINCLAIR et al., 2004; STRECK et al., 2005).

As variações dos QMe das equações de regressão linear simples para os diferentes valores de temperatura base usados no cálculo da soma térmica para A. montevidensis nas diferentes épocas de cultivo a campo foi em média 0,22 na faixa de Tb entre 0 e $10^{\circ} \mathrm{C}$. Nas épocas 1, 2, 3, 4 e 5, a variação do QMe entre plantas foi em média 0,054, 0,235, 0,204, 0,390 e 0,219, respectivamente. Os menores valores de QMe para todas as plantas dentro de cada época foi para Tb de $0^{\circ} \mathrm{C}$, apresentado valores de $0,426,0,219,0,130,0,129$ e 0,139 entre as épocas. Assim como preconizado no método (SINCLAIR et al., 2004), considerou-se como temperatura base para aparecimento de nós dessa espécie o valor de $0^{\circ} \mathrm{C}$. Não foram utilizados os dados coletados na época 6 , cultivo em casa de vegetação, pois as temperaturas foram elevadas e neste caso o método de estimativa de Tb não funciona (SINCLAIR et al., 2004).

Valores de $\mathrm{r}^{2}$ acima de 0,94 da regressão entre o NN na HP e STa foram obtidos em todas as épocas e em todas as hastes. Portanto, pode-se afirmar que o desenvolvimento de nós em $\boldsymbol{A}$. montevidensis é influenciado pela temperatura do ar como já foi reportado para outras espécies (BAKER \& REDDY, 2001; STRECK et al., 2005) e a linearidade entre NN e STa indica que a estimativa do plastocrono pelo método da regressão linear é uma metodologia adequada.
O plastocrono calculado variou entre as hastes e entre as épocas de cultivo (Tabela 1). Em geral, a HP foi a haste que apresentou os menores valores de

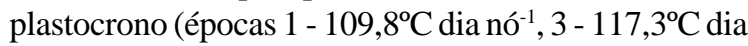
nó ${ }^{-1}$ e 6 - 148,6 ${ }^{\circ} \mathrm{C}$ dia nó ${ }^{-1}$ ), indicando que a emissão de nó e, conseqüentemente, a emissão de folhas é mais rápida na haste principal que nas demais hastes. Resultados semelhantes foram encontrados por PIVETTA et al. (2007) para o tomateiro, em que o filocrono da HP foi menor que nas hastes laterais. Em geral, as hastes de segunda ordem foram as que apresentaram os maiores valores de plastocrono, exceto na época 5, em que o menor valor de plastocrono $\left(145,3^{\circ} \mathrm{C}\right.$ dia nó $\left.{ }^{-1}\right)$ foi observado na HL2BB, época em que a altura das plantas foi menor. Talvez nesta época tenha havido um menor sombreamento dessa haste pelas demais, o que propiciou um desenvolvimento mais rápido se comparado às outras hastes. Com relação à posição na haste principal, a tendência foi de um menor plastocrono (maior velocidade de emissão de nós) nas hastes situadas na posição basal comparada à posição apical, no entanto, na maioria das épocas e das hastes laterais, a diferença foi tenha estatisticamente significativa (Tabela 1). A maior velocidade de emissão de nós nas hastes basais da haste principal pode estar associada à distribuição de fotoassimilados na planta, que pode resultar em maior disponibilidade de carboidratos e minerais na parte basal da haste principal. Esta hipótese, entretanto, necessita de estudos posteriores para ser confirmada.

Entre as épocas de cultivo, os menores valores de plastocrono foram observados nas épocas 1 , 2, e 3 e os maiores valores nas épocas 4 e 5 para a maioria das hastes analisadas (Tabela 1). Essas diferenças indicam que os maiores valores de plastocrono ocorreram nas épocas em que as

Tabela 1 - Plastocrono $\left({ }^{\circ} \mathrm{C}\right.$ dia nó $\left.{ }^{-1}\right)$ na haste principal (HP) e em hastes laterais de primeira (HL1B e HL1A ${ }^{\times}$) e segunda (HL2BB, HL2BA, HL2AB e HL2AA ${ }^{\times}$) ordem de Aspilia montevidensis. Efeito das hastes na vertical e efeito de épocas na horizontal. Santa Maria, RS, 2005/2006.

\begin{tabular}{llllllll}
\hline Haste & \multicolumn{1}{c}{ Época $1^{+}$} & \multicolumn{1}{c}{ Época 2} & \multicolumn{1}{c}{ Época 3} & Época 4 & \multicolumn{1}{c}{ Época 5} & Época 6 & CV (\%) \\
\hline HP & $109,8 \mathrm{cD}^{*}$ & $132,9 \mathrm{cdBC}$ & $117,3 \mathrm{cCD}$ & $194,5 \mathrm{aA}$ & $180,9 \mathrm{abA}$ & $148,6 \mathrm{bB}$ & 15,3 \\
HL1B & $142,9 \mathrm{bBC}$ & $127,5 \mathrm{dC}$ & $124,3 \mathrm{bcC}$ & $168,3 \mathrm{aAB}$ & $179,4 \mathrm{abA}$ & $155,0 \mathrm{abAB}$ & 19,3 \\
HL1A & $162,9 \mathrm{abABC}$ & $134,9 \mathrm{cdC}$ & $161,3 \mathrm{bcBC}$ & $199,5 \mathrm{aA}$ & $177,2 \mathrm{abAB}$ & $152,9 \mathrm{abBC}$ & 22,2 \\
HL2BB & $140,4 \mathrm{bA}$ & $172,0 \mathrm{abA}$ & $167,8 \mathrm{abcA}$ & $163,8 \mathrm{aA}$ & $145,3 \mathrm{bA}$ & $168,0 \mathrm{abA}$ & 20,5 \\
HL2BA & $151,9 \mathrm{abB}$ & $202,3 \mathrm{aA}$ & $179,5 \mathrm{abAB}$ & $181,6 \mathrm{aAB}$ & $188,9 \mathrm{aAB}$ & $174,2 \mathrm{aAB}$ & 21,4 \\
HL2AB & $164,9 \mathrm{bAB}$ & $149,6 \mathrm{bcdB}$ & $185,2 \mathrm{aAB}$ & $200,0 \mathrm{aA}$ & $200,0 \mathrm{aA}$ & $167,3 \mathrm{abAB}$ & 13,4 \\
HL2AA & $174,8 \mathrm{aA}$ & $162,4 \mathrm{bcA}$ & $192,3 \mathrm{aA}$ & $200,0 \mathrm{aA}$ & - & $161,6 \mathrm{abA}$ & 12,1 \\
CV (\%) & 13,5 & 17,7 & 23,2 & 21,9 & 20,4 & 14,9 & \\
\hline
\end{tabular}

*Médias não seguidas pela mesma letra minúscula na vertical e maiúscula na horizontal diferem entre si pelo teste DMS a 5\% de significância. Épocas de cultivo: 'época $1=(20 / 12 / 05)$, época $2=(14 / 02 / 06)$, época $3=(06 / 03 / 06)$, época $4=(04 / 04 / 06)$, época $5=$ $(05 / 05 / 06)$ e época $6=(08 / 06 / 06) .{ }^{\times} \mathrm{B}=$ basal, $\mathrm{A}=$ apical, $\mathrm{BB}=$ basal basal, $\mathrm{BA}=$ basal apical, $\mathrm{AB}=$ apical basal, $\mathrm{AA}=$ apical apical . 
temperaturas do ar foram mais baixas (épocas 4 e 5), diferindo do encontrado por CAO \& TIBBITS (1995) e PAULA et al. (2005), os quais verificaram que o filocrono (intervalo de tempo cronológico entre aparecimento de folhas sucessivas) em batata (Solanum tuberosum L.) aumenta com o aumento da temperatura do ar.

O NFN variou entre as hastes (Tabela 2). A tendência foi de um maior NFN na HP e de diminuição do NFN nas hastes laterais e à medida que aumentava o número de ordem da haste lateral. O NFN na HP diferiu estatisticamente das demais hastes nas épocas 1, 2 e 3, as quais apresentaram o maior NFN $(11,7,9,2$ e 7,4). O NFN da HP (7,5, 6,8 e 9,3) e HL1B (7,2, 6,2 e 8,8) nas épocas 4, 5 e 6 não diferiram entre si, mas diferiram das outras hastes. O menor NFN foi obtido na HL1A e nas hastes de segunda ordem. Com relação à posição na haste principal, a tendência foi de que hastes localizadas na base da haste principal apresentaram maior NFN, mas com diferença significativa apenas para as hastes laterais de primeira ordem (HL1). O NFN variou entre as épocas de cultivo (Tabela 2). Nos cultivos de campo, o atraso da época de cultivo diminuiu o NFN e as diferenças entre épocas foram maiores na HP e diminuíram nas hastes laterais, especialmente nas de segunda ordem (HL2). Diferenças de NFN entre hastes e épocas de cultivo e em relação à posição na haste principal podem estar relacionas com a partição de fotoassimilados, pois, segundo TAIZ \& ZEIGER (2004), ocorre uma distribuição diferencial de fotoassimilados pelos diferentes órgãos da planta.

Ao analisar conjuntamente a tendência dos valores de plastocrono e NFN nas diferentes hastes da planta, nota-se que as hastes com menor valor de plastocrono foram as que tiveram maior NFN (Tabelas
1 e 2). Assim, por exemplo, a HP teve menor plastocrono, ou seja, maior taxa de aparecimento de nós (e de folhas) e o maior NFN de todas as hastes. Esta relação inversa pode ser interpretada como uma estratégia da planta para garantir uma área foliar mínima antes de entrar na fase reprodutiva na haste principal. À medida que vão aparecendo hastes laterais na planta já existe uma área foliar mínima que garante a produção de fotoassimilados para o florescimento e o enchimento de grãos, o que pode contribuir para um menor NFN nas hastes laterais. $\mathrm{O}$ maior plastocrono (menor taxa de aparecimento de nós) nas hastes laterais pode ser o resultado da competição intra-específica por fotoassimilados na planta, haja vista que as hastes de menor ordem já estão na fase reprodutiva. No entanto, mais estudos deverão ser realizados para comprovar esta hipótese. Esta relação inversa entre plastocrono e NFN também caracteriza um sincronismo no desenvolvimento da planta de $\boldsymbol{A}$. montevidensis em direção à fase reprodutiva, já que um menor NFN antecipa o florescimento da haste. Este sincronismo é importante sob o ponto de vista de usar esta espécie para fins ornamentais e pode ser uma das características que o melhoramento deve considerar na domesticação desta espécie.

O NFN variou entre as épocas de cultivo (Tabela 2). Nos cultivos de campo, o atraso da época de cultivo diminuiu o NFN e as diferenças entre épocas foram maiores na HP e diminuíram nas hastes laterais, especialmente nas de segunda ordem (HL2). Assim, as épocas 1, 2 e 6 foram as épocas que apresentaram maior NFN na HP. Os menores valores de NFN foram observados nas épocas em que os valores de temperatura do ar eram menores. $\mathrm{O}$ fato de ter havido diferenças de NFN entre épocas pode ser um indicativo

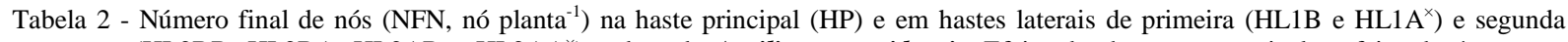
(HL2BB, HL2BA, HL2AB e HL2AA ${ }^{\times}$) ordem de Aspilia montevidensis. Efeito das hastes na vertical e efeito de épocas na horizontal. Santa Maria, RS, 2005/2006.

\begin{tabular}{llllllll}
\hline Hastes & ${\text { Época } 1^{+}}^{+}$ & Época 2 & Época 3 & Época 4 & Época 5 & Época 6 & CV (\%) \\
\hline HP & $11,7 \mathrm{aA} *$ & $9,2 \mathrm{aB}$ & $7,4 \mathrm{aC}$ & $7,5 \mathrm{aC}$ & $6,8 \mathrm{aC}$ & $9,3 \mathrm{aB}$ & 18,1 \\
HL1B & $5,4 \mathrm{bCD}$ & $4,8 \mathrm{bD}$ & $5,8 \mathrm{bCD}$ & $7,2 \mathrm{aB}$ & $6,2 \mathrm{aBC}$ & $8,8 \mathrm{aA}$ & 22,6 \\
HL1A & $3,4 \mathrm{cA}$ & $2,9 \mathrm{cABC}$ & $2,5 \mathrm{dBC}$ & $2,3 \mathrm{bC}$ & $2,6 \mathrm{bBC}$ & $3,2 \mathrm{bcAB}$ & 26,6 \\
HL2BB & $3,0 \mathrm{cAB}$ & $1,7 \mathrm{cC}$ & $3,7 \mathrm{cAB}$ & $3,7 \mathrm{bAB}$ & $2,4 \mathrm{bBC}$ & $4,0 \mathrm{bA}$ & 39,8 \\
HL2BA & $2,3 \mathrm{cAB}$ & $2,3 \mathrm{cAB}$ & $1,9 \mathrm{deB}$ & $2,3 \mathrm{bAB}$ & $2,0 \mathrm{bAB}$ & $2,8 \mathrm{cA}$ & 35,7 \\
HL2AB & $2,6 \mathrm{cA}$ & $2,8 \mathrm{cA}$ & $1,5 \mathrm{deA}$ & $2,0 \mathrm{bA}$ & $2,0 \mathrm{bA}$ & $2,3 \mathrm{cA}$ & 39,9 \\
HL2AA & $2,3 \mathrm{cA}$ & $1,8 \mathrm{cA}$ & $1,4 \mathrm{eA}$ & $2,0 \mathrm{bA}$ & $1,0 \mathrm{bA}$ & $2,6 \mathrm{cA}$ & 44,6 \\
CV (\%) & 24,5 & 29,2 & 25,2 & 31,4 & 28.6 & 24,8 & \\
\hline
\end{tabular}

*Médias não seguidas pela mesma letra minúscula na vertical e maiúscula na horizontal diferem entre si pelo teste DMS a 5\% de significância. CV = Coeficiente de variação. Épocas de cultivo: ${ }^{+}$época $1=(20 / 12 / 05)$, época $2=(14 / 02 / 06)$, época $3=(06 / 03 / 06)$, época $4=$ $(04 / 04 / 06)$, época $5=(05 / 05 / 06)$ e época $6=(08 / 06 / 06) .{ }^{\times} \mathrm{B}=$ basal, $\mathrm{A}=$ apical, BB $=$ basal basal, BA $=$ basal apical, AB = apical basal, AA = apical apical. 
de que o desenvolvimento desta espécie seja afetado pelo fotoperíodo, já que o NFN é um indicador morfológico da resposta fotoperiódica de cultivos agrícolas anuais como o trigo e o arroz (STRECK et al., 2003; STRECK et al., 2006b).

\section{CONCLUSÕES}

A temperatura base estimada para emissão de nós de $\boldsymbol{A}$. montevidensis foi de $0^{\circ} \mathrm{C}$. O plastocrono e o número final de nós variam entre hastes e épocas de cultivo, com os menores valores de plastocrono e maiores valores de número final de nós observados na haste principal.

\section{AGRADECIMENTOS}

Os autores agradecem à Coordenação de Aperfeiçoamento de Pessoal de Nível Superior (CAPES), pela bolsa de Mestrado e ao Programa de Pós-graduação em Agronomia da UFSM pelo apoio.

\section{REFERÊNCIAS}

ARNOLD, C.Y. Maximum-minimum temperature as a basis for computing heat units. Proceedings of the American Society for Horticultural Science, v.76, p.682-692, 1960.

BAKER, J.T.; REDDY, V.R. Temperature effects on phenological development and yield of muskmelon. Annals of Botany, v.87, n.5, p.605-613, 2001.

BELLINI, A.A. et al. Steroidal saponin, 7-oxostigmasterol and diterpenes from Aspilia montevidensis. Biochemical Systematics and Ecology, v.27, p.317-319, 1999.

BRUNINI, O. et al. Temperatura base para alface "White Boston”, em um sistema de unidades térmicas. Bragantia, v.35, n.1, p.214-219, 1976.

CAO, W.; TIBBITTS, T.W. Leaf emergence on potato stems in relation to thermal time. Agronomy Journal, v.87, n.3, p.474-477, 1995.

EMBRAPA. Ambiente de software NTIA, versão 4.2.2 manual do usuário. Campinas: Centro Nacional de Pesquisa Tecnológica em Informática para a Agricultura, 1997. 258p.

GILMORE Jr., E.C.; ROGERS, J.S. Heat units as a method of measuring maturity in corn. Agronomy Journal, v.50, n.10, p.611-615, 1958

GRAMBONE-GUARATINI, M.T. et al. Reproductive biology in especies of Bidens L. (Asteraceae). Scientia Agricola, v.61, n.2, p.185-189, 2004.

HODGES, T. Introduction. In: HODGES, T. Predicting crop phenology. Boston: CRC, 1991. p.1-2.
KISSMANN, K.G.; GROTH, D. Plantas infestantes e nocivas. 2.ed. São Paulo: BASF, 1999. T.II, p.199-203.

McMASTER, G.S.; SMIKA, D.E. Estimation and evaluation of winter wheat phenology in the central Great Plains. Agricultural and Forest Meteorology, v.43, n.1, p.1-8, 1988.

McMASTER, G.S.; WILHELM, W.W. Growing degree-days: one equation, two interpretations. Agricultural and Forest Meteorology, v.87, n.4, p.291-300, 1997

PAULA, F.L.M. et al. Filocrono da planta de batata cultivar Asterix em diferentes épocas de plantio. Revista Brasileira de Agrometeorologia, v.13, n.3, p.367-374, 2005.

PEREIRA, A.R. et al. Agrometeorologia: fundamentos e aplicações práticas. Guaíba: Agropecuária, 2002. 478p.

PIVETTA, C.R. et al. Emissão e expansão foliar em três genótipos de tomateiro (Lycopersicon esculentum Mill.). Ciência Rural, v.37, n.5, p.1274-1280, 2007.

RUSSELE, M.P. et al. Growth analysis based on degree days. Crop Science, v.24, n.1, p.28-32, 1984.

SIMÕES, C.M.O. et al. Plantas da medicina popular no Rio Grande do Sul. 3.ed. Porto Alegre: UFRGS, 1989. 174p.

SINCLAIR, T.R. Leaf area development in field grow soybean. Agronomy Journal, v.76, n.1, p.141-146, 1984

SINCLAIR, T.R. et al. Sugarcane leaf area development under field conditions in Florida, USA. Field crop Research, v.88, n.1, p.171-178, 2004

STRECK, N.A. et al. Improving predictions of developmental stages in winter wheat: a modified Wang and Engel model. Agricultural and Forest Meteorology, v.115, n.3-4, p.139150, 2003.

STRECK, N.A. et al. Estimativa do plastocrono em meloeiro (Cucumis melo L.) cultivado em estufa plástica em diferentes épocas do ano. Ciência Rural, v.35, n.6, p.1275-1280, 2005.

STRECK, N.A. et al. Duração do ciclo de desenvolvimento de cultivares de arroz em função da emissão de folhas no colmo principal. Ciência Rural, v.36, n.4, p.1086-1093, 2006a.

STRECK, N.A. et al. Avaliação da resposta ao fotoperíodo em genótipos de arroz irrigado. Bragantia, v.65, n.4, p.533-541, 2006b.

TAIZ, L.; ZEIGER, E. Fisiologia vegetal. 3.ed. Porto Alegre: Artmed, 2004. 719p.

WILHELM, W.W.; McMASTER, G.S. Importance of the phyllochron in studying development and growth in grasses. Crop Science, v.35, n.1, p.1-3, 1995. 